\title{
Fixed Point Results Satisfying Rational Type Contraction in G-Metric Spaces
}

\author{
Branislav Z. Popović, ${ }^{1}$ Muhammad Shoaib, ${ }^{2}$ and Muhammad Sarwar ${ }^{2}$ \\ ${ }^{1}$ Faculty of Science, University of Kragujevac, Radoja Domanovića 12, 34000 Kragujevac, Serbia \\ ${ }^{2}$ Department of Mathematics, University of Malakand, Chakdara, Lower Dir 18800, Pakistan \\ Correspondence should be addressed to Branislav Z. Popović; bpopovic@kg.ac.rs
}

Received 15 May 2016; Accepted 6 June 2016

Academic Editor: Filomena Cianciaruso

Copyright (c) 2016 Branislav Z. Popović et al. This is an open access article distributed under the Creative Commons Attribution License, which permits unrestricted use, distribution, and reproduction in any medium, provided the original work is properly cited.

A unique fixed point theorem for three self-maps under rational type contractive condition is established. In addition, a unique fixed point result for six continuous self-mappings through rational type expression is also discussed.

\section{Introduction}

Fixed point theory is one of the core subjects of nonlinear analysis. This theory is not constrained to mathematics; it is also applicable to other disciplines. It is closely linked with social and medical science, military applications, graph theory [1], game theory, economics [2], statistics, and medicine. This theory is divided into three categories: topological fixed point theory, metric fixed point theory, and discrete fixed point theory.

In metric fixed point theory, the first result proved by Banach [3] is known as Banach contraction principle. Many researchers extended this principle for the study of fixed points and common fixed points using different types of contraction such as weak contraction $[4,5]$, integral type contraction [6], rational type contraction [7], and T-Hardy Rogers type contraction [8]. For more details, see [9-11] and so forth.

Dass and Gupta [12] gave the extension of Banach's contraction mapping principle by using a contractive condition of rational type. Jaggi [7] proved some unique fixed point results through contractive condition of rational type in metric spaces. Harjani et al. [13] studied the results of Jaggi in the setting of partially ordered metric spaces. Using generalized weak contractions Luong and Thuan [14] generalized the results of [13] through rational type expressions in the context of partially ordered metric spaces. Chandok and Karapinar [15] generalized the results of Harjani and established common fixed point results for weak contractive conditions satisfying rational type expressions in partially ordered metric spaces. Mustafa et al. [16] discussed fixed point results by almost generalized contraction via rational type expression which generalizes, extends, and unifies the results of Jaggi [7], Harjani et al. [13], and Luong and Thuan [14], respectively. Fixed point theorems for contractive type conditions satisfying rational inequalities in metric spaces have been developed in a number of works; see [17-20] and so forth.

Mustafa and Sims [21] generalized the notion of metric space as an appropriate notion of generalized metric space called $G$-metric space. They have investigated convergence in $G$-metric spaces, introduced completeness of $G$-metric spaces, and proved a Banach contraction mapping theorem and some other fixed point theorems involving contractive type mappings in $G$-metric spaces using different contractive conditions. Later, various authors have proved some common fixed point theorems in these spaces (see [8, 22-24]).

Sanodia et al. [25] used rational type contraction and investigated a unique fixed point theorem for single mapping in $G$-metric spaces. Gandhi and Bajpai [26] generalized the result of Sanodia et al. and proved unique common fixed point results for three mappings in $G$-metric space satisfying 
rational type contractive condition. Recently, Shrivastava et al. [27] established some unique fixed point theorem for some new rational type contraction.

The aim of this paper is to establish two common fixed point theorems satisfying rational type contraction. In the first result, we discuss the existence and uniqueness of common fixed point for three self-maps in the context of $G$-metric space, while in the second one we studied the uniqueness of common fixed point for six continuous selfmappings in the setting of $G$-metric through rational type expression.

\section{Preliminaries}

We recall some definitions that will be used in our discussion.

Definition 1 (see [21]). Let $X$ be a nonempty set and let $G: X \times$ $X \times X \rightarrow \mathbb{R}^{+}$be a function satisfying the following conditions:

(1) $G(x, y, z)=0$ implies that $x=y=z$ for all $x, y, z \in$ $X$.

(2) $G(x, x, y) \leq G(x, y, z)$ for all $x, y, z \in X$.

(3) $G(x, y, z)=G(x, z, y)=G(y, z, x) \cdots$ for all $x, y, z \in$ $X$.

(4) $G(x, y, z) \leq G(x, a, a)+G(a, y, z)$ for all $x, y, z, a \in X$.

Then, it is called $G$-metric and the pair $(X, G)$ is a $G$ metric space.

Proposition 2 (see [21]). Let $(X, G)$ be a $G$-metric space. The following are equivalent:

(1) $\left(x_{n}\right)$ is G-convergent to $x$.

(2) $G\left(x_{n}, x_{n}, x\right) \rightarrow 0$ as $n \rightarrow \infty$.

(3) $G\left(x_{n}, x, x\right) \rightarrow 0$ as $n \rightarrow \infty$.

(4) $G\left(x_{n}, x_{m}, x\right) \rightarrow 0$ as $n, m \rightarrow \infty$.

Definition 3 (see $[22,28]$ ). A pair of self-mappings $f, g$ in a $G$-metric space is said to be weakly commuting if

$$
G(f g x, g f x, g f x) \leq G(f x, g x, g x), \quad \forall x \in X
$$

Sanodia et al. [25] proved the following fixed point theorem in the setting of $G$-metric space.

Theorem 4. Let $(X, G)$ be a $G$-complete $G$-metric space and let $f: X \rightarrow X$ be a self-map satisfying the condition

$$
\begin{aligned}
& G(f x, f y, f z) \leq A \\
& \quad \cdot \frac{\max \left\{G^{2}(x, f x, f y), G^{2}(y, f y, f z), G^{2}(z, f z, f x)\right\}}{G(x, y, z)}
\end{aligned}
$$

for all $x, y, z \in X$ with $0 \leq A<1$. Then, $f$ has a unique common fixed point in $X$.
Theorem 5. Let $(X, G)$ be a G-complete $G$-metric space and let $S, T: X \rightarrow X$ be two self-maps such that $S(X) \subset T(X)$ satisfying the following condition:

$$
\begin{aligned}
& G(T x, T y, T z) \leq A \\
& \quad \cdot \frac{\max \left\{G^{2}(S x, T x, T y), G^{2}(S y, T y, T z), G^{2}(S z, T z, T x)\right\}}{G(S x, S y, S z)}
\end{aligned}
$$

for all $x, y, z \in X$ with $0 \leq A<1$. Then, $S$ and T have a unique common fixed point in $X$.

Gandhi and Bajpai [26] proved unique common fixed point results satisfying the following rational type contractive condition.

Theorem 6. Let $(X, G)$ be a $G$-complete $G$-metric space and let $f, g, h: X \rightarrow X$ be three self-mappings satisfying the condition

$$
\begin{aligned}
& G(f x, g y, h z) \leq A \\
& \quad \frac{\max \left\{G^{2}(x, f x, g y), G^{2}(y, g y, h z), G^{2}(z, h z, f x)\right\}}{G(x, y, z)}
\end{aligned}
$$

for all $x, y, z \in X$ with $0 \leq A<1$. Then, $f, g$, and $h$ have $a$ unique common fixed point in $X$.

Currently, Shrivastava et al. [27] studied the following result.

Theorem 7. Let $(X, G)$ be a $G$-complete $G$-metric space and let $f: X \rightarrow X$ be a self-map satisfying the condition

$$
\begin{aligned}
& G(f x, f y, f z) \leq A \cdot \frac{G(x, f y, f y)+G(x, f z, f z)}{2}+B \\
& \cdot(G(x, f y, f y) G(x, f y, f y)+G(x, f z, f z) \\
& \quad+G(y, f x, f x)+G(z, f x, f x)) \\
& \quad \cdot(2(G(x, f y, f y)+G(y, f x, f x)))^{-1}
\end{aligned}
$$

for all $x, y, z \in X$ with $0 \leq A+B<1 / 2$. Then, $f$ has a unique common fixed point in $X$ and $f$ is G-continuous at $u$.

\section{Main Results}

Our first new result is the following.

Theorem 8. Let $(X, G)$ be a $G$-complete $G$-metric space and let $S, T, R: X \rightarrow X$ be three self-mappings satisfying the following condition:

$$
\begin{aligned}
& G(S x, T y, R z) \leq A \cdot(G(x, S x, T y) G(y, T y, R z) \\
& \left.\quad+[G(x, y, z)]^{2}+G(x, S x, T y) G(x, y, z)\right) \\
& \quad \cdot(G(x, S x, T y)+G(x, y, z)+G(y, T y, R z))^{-1} \\
& \quad+B \cdot(G(y, T y, R z)[1+G(x, S x, T y)] \\
& \left.\quad \cdot(1+G(x, y, z))^{-1}\right)+C \cdot G(x, y, z)
\end{aligned}
$$


for all $x, y, z \in X$ with $x \neq y \neq z \neq x, A, B, C \geq 0$ with $0 \leq A+$ $B+C<1, G(x, S x, T y)+G(x, y, z)+G(x, T y, R z) \neq 0$. Then, $S$, $T$, and $R$ have a common fixed point. Further, if $G(x, S x, T y)+$ $G(x, y, z)+G(x, T y, R z)=0$ implies $G(S x, T y, R z)=0$, then $S, T$, and $R$ have a unique common fixed point in $X$.

Proof. Let $x_{0}$ be arbitrary in $X$; we define a sequence $x_{n}$ by the following rules:

$$
\begin{aligned}
& x_{3 n+1}=S x_{3 n}, \\
& x_{3 n+2}=T x_{3 n+1}, \\
& x_{3 n+3}=R x_{3 n+2},
\end{aligned}
$$

\section{$\forall n \in \mathbb{X}$}

Now, we have to show that $x_{n}$ is a $G$-Cauchy sequence in $X$. Consider $G(x, S x, T y)+G(x, y, z)+G(x, T y, R z) \neq 0$; from (6), we have

$$
\begin{aligned}
& G\left(x_{3 n+1}, x_{3 n+2}, x_{3 n+3}\right)=G\left(S x_{3 n}, T x_{3 n+1}, R x_{3 n+2}\right) \leq A \\
& \cdot\left[G\left(x_{3 n}, S x_{3 n}, T x_{3 n+1}\right) G\left(x_{3 n+1}, T x_{3 n+1}, R x_{3 n+2}\right)\right. \\
& +\left[G\left(x_{3 n}, x_{3 n+1}, x_{3 n+2}\right)\right]^{2}+G\left(x_{3 n}, S x_{3 n}, T x_{3 n+1}\right) \\
& \left.\cdot G\left(x_{3 n}, x_{3 n+1}, x_{3 n+2}\right)\right]\left(G\left(x_{3 n}, S x_{3 n}, T x_{3 n+1}\right)\right. \\
& +G\left(x_{3 n}, x_{3 n+1}, x_{3 n+2}\right) \\
& \left.+G\left(x_{3 n+1}, T x_{3 n+1}, R x_{3 n+2}\right)\right)^{-1}+B \\
& \cdot\left(G\left(x_{3 n+1}, T x_{3 n+1}, R x_{3 n+2}\right)[1\right. \\
& \left.+G\left(x_{3 n}, S x_{3 n}, T x_{3 n+1}\right)\right](1 \\
& \left.\left.+G\left(x_{3 n}, x_{3 n+1}, x_{3 n+2}\right)\right)^{-1}\right)+C \cdot G\left(x_{3 n}, x_{3 n+1}, x_{3 n+2}\right) \\
& =A \cdot\left[G\left(x_{3 n}, x_{3 n+1}, x_{3 n+2}\right) G\left(x_{3 n+1}, x_{3 n+2}, x_{3 n+3}\right)\right. \\
& +\left[G\left(x_{3 n}, x_{3 n+1}, x_{3 n+2}\right)\right]^{2}+G\left(x_{3 n}, x_{3 n+1}, x_{3 n+2}\right) \\
& \left.\cdot G\left(x_{3 n}, x_{3 n+1}, x_{3 n+2}\right)\right]\left(G\left(x_{3 n}, x_{3 n+1}, x_{3 n+2}\right)\right. \\
& \left.+G\left(x_{3 n}, x_{3 n+1}, x_{3 n+2}\right)+G\left(x_{3 n+1}, x_{3 n+2}, x_{3 n+3}\right)\right)^{-1} \\
& +B \cdot\left(G\left(x_{3 n+1}, x_{3 n+2}, x_{3 n+3}\right)[1\right. \\
& \left.+G\left(x_{3 n}, x_{3 n+1}, x_{3 n+2}\right)\right](1 \\
& \left.\left.+G\left(x_{3 n}, x_{3 n+1}, x_{3 n+2}\right)\right)^{-1}\right)+C \cdot G\left(x_{3 n}, x_{3 n+1}, x_{3 n+2}\right) \\
& =A \cdot\left[G ( x _ { 3 n } , x _ { 3 n + 1 } , x _ { 3 n + 2 } ) \left(G\left(x_{3 n+1}, x_{3 n+2}, x_{3 n+3}\right)\right.\right. \\
& \left.\left.+G\left(x_{3 n}, x_{3 n+1}, x_{3 n+2}\right)+G\left(x_{3 n}, x_{3 n+1}, x_{3 n+2}\right)\right)\right] \\
& \cdot\left(G\left(x_{3 n}, x_{3 n+1}, x_{3 n+2}\right)+G\left(x_{3 n}, x_{3 n+1}, x_{3 n+2}\right)\right. \\
& \left.+G\left(x_{3 n+1}, x_{3 n+2}, x_{3 n+3}\right)\right)^{-1}+B \\
& \cdot\left(G\left(x_{3 n+1}, x_{3 n+2}, x_{3 n+3}\right)\left[1+G\left(x_{3 n}, x_{3 n+1}, x_{3 n+2}\right)\right]\right.
\end{aligned}
$$

$$
\begin{aligned}
& \left.\cdot\left(1+G\left(x_{3 n}, x_{3 n+1}, x_{3 n+2}\right)\right)^{-1}\right)+C \cdot G\left(x_{3 n}, x_{3 n+1},\right. \\
& \left.x_{3 n+2}\right)=A \cdot G\left(x_{3 n}, x_{3 n+1}, x_{3 n+2}\right)+B \cdot G\left(x_{3 n+1},\right. \\
& \left.x_{3 n+2}, x_{3 n+3}\right)+C \cdot G\left(x_{3 n}, x_{3 n+1}, x_{3 n+2}\right)=(A+C) \\
& \cdot G\left(x_{3 n}, x_{3 n+1}, x_{3 n+2}\right)+B \cdot G\left(x_{3 n+1}, x_{3 n+2}, x_{3 n+3}\right),
\end{aligned}
$$

which implies that

$$
G\left(x_{3 n+1}, x_{3 n+2}, x_{3 n+3}\right) \leq h \cdot G\left(x_{3 n}, x_{3 n+1}, x_{3 n+2}\right),
$$

where $h=(A+C) /(1-B)$.

Similarly,

$$
G\left(x_{3 n+3}, x_{3 n+4}, x_{3 n+5}\right) \leq h \cdot G\left(x_{3 n+2}, x_{3 n+3}, x_{3 n+4}\right) \text {. }
$$

Therefore, for all $n$, we have

$$
\begin{aligned}
G\left(x_{n+1}, x_{n+2}, x_{n+3}\right) & \leq h \cdot G\left(x_{n}, x_{n+1}, x_{n+2}\right) \leq \cdots \\
& \leq h^{n+1} \cdot G\left(x_{0}, x_{1}, x_{2}\right) .
\end{aligned}
$$

Now, for all $l, m, n$, with $l>m>n$, using rectangular inequality, the second axiom of the G-metric, and (11), we have

$$
\begin{aligned}
G\left(x_{n}, x_{m}, x_{l}\right) \leq & G\left(x_{n}, x_{n+1}, x_{n+1}\right) \\
& +G\left(x_{n+1}, x_{n+2}, x_{n+2}\right)+\cdots \\
& +G\left(x_{l-2}, x_{l-1}, x_{l}\right) \\
\leq & G\left(x_{n}, x_{n+1}, x_{n+2}\right) \\
& +G\left(x_{n+1}, x_{n+2}, x_{n+3}\right)+\cdots \\
& +G\left(x_{l-2}, x_{l-1}, x_{l}\right) \\
\leq & h^{n}+h^{n+1}+\cdots+h^{l-2} \cdot G\left(x_{0}, x_{1}, x_{2}\right) \\
= & \frac{h^{n}}{1-h} \cdot G\left(x_{0}, x_{1}, x_{2}\right),
\end{aligned}
$$

where $G\left(x_{n}, x_{m}, x_{l}\right) \rightarrow 0$ as $n, m, l \rightarrow \infty$.

This shows that $x_{n}$ is a $G$-Cauchy sequence. But $(X, G)$ is $G$-complete $G$-metric space so there exists $w$ in $X$ such that $x_{n} \rightarrow w$ as $n$ tends to infinity.

Now, we assume that $s w \neq w$. Using condition (6), we have

$$
\begin{aligned}
& G\left(S w, x_{3 n+2}, x_{3 n+3}\right)=G\left(S w, T x_{3 n+1}, R x_{3 n+2}\right) \leq A \\
& \quad \cdot\left[G\left(w, S w, T x_{3 n+1}\right) G\left(x_{3 n+1}, T x_{3 n+1}, R x_{3 n+2}\right)\right. \\
& \quad+\left[G\left(w, x_{3 n+1}, x_{3 n+2}\right)\right]^{2}+G\left(w, S w, T x_{3 n+1}\right) \\
& \left.\quad \cdot G\left(w, x_{3 n+1}, x_{3 n+2}\right)\right]\left(G\left(w, S w, T x_{3 n+1}\right)\right. \\
& \quad+G\left(w, x_{3 n+1}, x_{3 n+2}\right) \\
& \left.\quad+G\left(x_{3 n+1}, T x_{3 n+1}, R x_{3 n+2}\right)\right)^{-1}+B
\end{aligned}
$$




$$
\begin{aligned}
& \cdot\left(G\left(x_{3 n+1}, T x_{3 n+1}, R x_{3 n+2}\right)\right. \\
& \cdot\left[1+G\left(w, S w, T x_{3 n+1}\right)\right] \\
& \left.\cdot\left(1+G\left(w, x_{3 n+1}, x_{3 n+2}\right)\right)^{-1}\right)+C \\
& \cdot G\left(w, x_{3 n+1}, x_{3 n+2}\right)=A \cdot\left[G\left(w, S w, x_{3 n+2}\right)\right. \\
& \cdot G\left(x_{3 n+1}, x_{3 n+2}, x_{3 n+3}\right)+\left[G\left(w, x_{3 n+1}, x_{3 n+2}\right)\right]^{2} \\
& \left.+G\left(w, S w, x_{3 n+2}\right) G\left(w, x_{3 n+1}, x_{3 n+2}\right)\right] \\
& \cdot\left(G\left(w, s w, x_{3 n+2}\right)+G\left(w, x_{3 n+1}, x_{3 n+2}\right)\right. \\
& \left.+G\left(x_{3 n+1}, x_{3 n+2}, x_{3 n+3}\right)\right)^{-1}+B \\
& \cdot\left(G\left(x_{3 n+1}, x_{3 n+2}, x_{3 n+3}\right)\left[1+G\left(w, S w, x_{3 n+2}\right)\right]\right. \\
& \left.\cdot\left(1+G\left(w, x_{3 n+1}, x_{3 n+2}\right)\right)^{-1}\right)+C \\
& \cdot G\left(w, x_{3 n+1}, x_{3 n+2}\right) .
\end{aligned}
$$

As $x_{n}$ is $G$-Cauchy sequence and converges to $w$, therefore, by taking limit $n \rightarrow \infty$, we get $G(S w, w, w) \leq 0$ which is held only if $G(S w, w, w)=0$ implies that $S w=w$. Similarly, it can be shown that $T w=w$ and $R w=w$. Hence, $w$ is a common fixed point of $S, T$ and $R$.

Uniqueness. Suppose that $S, T$, and $R$ have two common fixed points $z$ and $w$ such that $z \neq w$. Since condition $G(x, S x, T y)+$ $G(x, y, z)+G(x, T y, R z)=0$ implies $G(S x, T y, R z)=0$, we have that $G(z, S z, T w)+G(z, w, w)+G(z, T w, R w)=0$ implies $G(S z, T w, R w)=0$. Therefore, one can get the following:

$$
G(S z, T w, R w)=G(z, w, w)=0
$$

$$
\text { implies that } z=w \text {, }
$$

which is a contradiction. Therefore, the common fixed point is unique.

Corollary 9. Let $(X, G)$ be a $G$-complete $G$-metric space and let $S, T, R: X \rightarrow X$ be three self-mappings satisfying the condition

$$
\begin{aligned}
& G(S x, T y, R z) \leq A \cdot[G(x, S x, T y) G(x, T y, R z) \\
& \left.\quad+[G(x, y, z)]^{2}+G(x, S x, T y) G(x, y, z)\right] \\
& \quad \cdot(G(x, S x, T y)+G(x, y, z)+G(x, T y, R z))^{-1}
\end{aligned}
$$

for all $x, y, z \in X$ with $x \neq y \neq z \neq x A \geq 0$ with $0 \leq A<$ $1, G(x, S x, T y)+G(x, y, z)+G(x, T y, R z) \neq 0$. Then, $S, T$, and $R$ have a common fixed point. Further, if $G(x, S x, T y)+$ $G(x, y, z)+G(x, T y, R z)=0$ implies $G(S x, T y, R z)=0$, then $S, T$, and $R$ have a unique common fixed point in $X$.

Proof. The proof follows by taking $B=C=0$ in Theorem 8 .
Corollary 10. Let $(X, G)$ be a $G$-complete $G$-metric space and let $S, T, R: X \rightarrow X$ be three self-mappings satisfying the condition

$$
\begin{aligned}
G(S x, T y, R z) \leq & B \\
& \cdot \frac{G(y, T y, R z)[1+G(x, S x, T y)]}{1+G(x, y, z)} \\
& +C \cdot G(x, y, z)
\end{aligned}
$$

for all $x, y, z \in X$ with $x \neq y \neq z \neq x B, C \geq 0$ with $0 \leq$ $B+C<1, G(x, S x, T y)+G(x, y, z)+G(x, T y, R z) \neq 0$. Then $S$, $T$, and $R$ have a common fixed point. Further, if $G(x, S x, T y)+$ $G(x, y, z)+G(x, T y, R z)=0$ implies $G(S x, T y, R z)=0$, then $S, T$, and $R$ have a unique common fixed point in $X$.

Proof. The proof follows by taking $A=0$ in Theorem 8 .

Corollary 11. Let $(X, G)$ be a $G$-complete $G$-metric space and let $S, T: X \rightarrow X$ be two self-mappings satisfying the condition

$$
\begin{aligned}
G(S x, T y, T z) \leq A \cdot[G(x, S x, T y) G(x, T y, T z) \\
\left.\quad+[G(x, y, z)]^{2}+G(x, S x, T y) G(x, y, z)\right] \\
\quad \cdot(G(x, S x, T y)+G(x, y, z)+G(x, T y, T z))^{-1} \\
\quad+B \cdot(G(y, T y, T z)[1+G(x, S x, T y)] \\
\left.\quad \cdot(1+G(x, y, z))^{-1}\right)+C \cdot G(x, y, z)
\end{aligned}
$$

for all $x, y, z \in X$ with $x \neq y \neq z \neq x A, B, C \geq 0$ with $0 \leq A+$ $B+C<1, G(x, S x, T y)+G(x, y, z)+G(x, T y, T z) \neq 0$. Then, $S$ and $T$ have a common fixed point. Further, if $G(x, S x, T y)+$ $G(x, y, z)+G(x, T y, T z)=0$ implies $G(S x, T y, T z)=0$, then $S$ and $T$ have a unique common fixed point in $X$.

Proof. The proof follows by taking $R=T$ in Theorem 8 .

By setting $R=T=S$ in Theorem 8 , we have the following corollary.

Corollary 12. Let $(X, G)$ be a $G$-complete $G$-metric space and let $T: X \rightarrow X$ be a self-mapping satisfying the condition

$$
\begin{aligned}
G & (T x, T y, T z) \leq A \cdot[G(x, T x, T y) G(x, T y, T z) \\
& \left.+[G(x, y, z)]^{2}+G(x, T x, T y) G(x, y, z)\right] \\
& \cdot(G(x, T x, T y)+G(x, y, z)+G(x, T y, T z))^{-1} \\
& +B \cdot(G(y, T y, T z)[1+G(x, T x, T y)] \\
& \left.\cdot(1+G(x, y, z))^{-1}\right)+C \cdot G(x, y, z)
\end{aligned}
$$

for all $x, y, z \in X$ with $x \neq y \neq z \neq x A, B, C \geq 0$ with $0 \leq A+B+C<1, G(x, T x, T y)+G(x, y, z)+G(x, T y, T z) \neq 0$. Then, $T$ has a unique fixed point. Further, if $G(x, T x, T y)+$ $G(x, y, z)+G(x, T y, T z)=0$ implies $G(T x, T y, T z)=0$, then $T$ has a unique common fixed point in $X$. 
The second main result in this section is the following.

Theorem 13. Let $(X, G)$ be a $G$-complete $G$-metric space. Let $R, S, T, I, J, Q: X \rightarrow X$ be six continuous self-maps and let $\{S, I\},\{T, J\}$, and $\{R, Q\}$ be weakly commuting pairs of selfmapping such that $T(X) \subset I(X), S(X) \subset J(X)$, and $R(X) \subset$ $Q(X)$, satisfying the condition

$$
\begin{aligned}
G( & R x, S y, T z) \leq A \cdot[G(Q x, S x, I z) G(R x, S x, I x) \\
& +[G(Q x, J y, I z)]^{2} \\
& +G(R x, S x, I x) G(Q x, J y, I z)](G(R x, S x, I x) \\
& +G(Q x, J y, I z)+G(R x, S x, I x))^{-1}+B \\
& +G(Q x, J y, I z)
\end{aligned}
$$

for all $x, y, z \in X$ with $x \neq y \neq z \neq x A, B \geq 0$ with $0 \leq A+B<1, G(R x, S x, I x)+G(Q x, J y, I z)+G(R x, S x, I x) \neq$ 0 . Then $R, S, T, I, J, Q$ have a common fixed point. Further, if $G(R x, S x, I x)+G(Q x, J y, I z)+G(R x, S x, I x)=0$ implies $G(S x, T y, R z)+G(Q x, J y, I z)=0$, then $R, S, T, I, J, Q$ have a unique common fixed point in $X$.

Proof. Take $x_{0}$ as arbitrary point of $X$. Since $R(X) \subset Q(X)$, we can find a point $x_{1}$ in $X$ such that $R x_{0}=Q x_{1}$. For $S(X) \subset J(X)$, we can find a point $x_{2}$ in $X$ such that $R x_{1}=Q x_{2}$ and for $T(X) \subset I(X)$ we can find a point $x_{3}$ in $X$ such that $T x_{2}=I x_{3}$. Generally, for a point $x_{3 n}$, choose $x_{3 n+1}$ such that $R x_{3 n}=Q x_{3 n+1}$; for a point $x_{3 n+1}$, choose $x_{3 n+2}$ such that $S x_{3 n+1}=J x_{3 n+2}$; and for a point $x_{3 n+2}$, choose $x_{3 n+3}$ such that $T x_{3 n+2}=I x_{3 n+3}$ for $n=0,1,2,3, \ldots$

Suppose $G_{3 n}=G\left(R x_{3 n}, S x_{3 n+1}, T x_{3 n+2}\right) \neq 0$ and $G_{3 n+1}=$ $G\left(R x_{3 n+1}, S x_{3 n+2}, T x_{3 n+3}\right) \neq 0$. Then, from condition (19), we have

$$
\begin{aligned}
G_{3 n+1} & =G\left(R x_{3 n+1}, S x_{3 n+2}, T x_{3 n+3}\right) \leq A \\
\cdot & {\left[G\left(Q x_{3 n+1}, S x_{3 n+1}, I x_{3 n+3}\right)\right.} \\
\cdot & G\left(R x_{3 n+1}, S x_{3 n+1}, I x_{3 n+1}\right) \\
+ & {\left[G\left(Q x_{3 n+1}, J x_{3 n+2}, I x_{3 n+3}\right)\right]^{2} } \\
+ & G\left(R x_{3 n+1}, S x_{3 n+1}, I x_{3 n+1}\right) \\
\cdot & \left.G\left(Q x_{3 n+1}, J x_{3 n+2}, I x_{3 n+3}\right)\right] \\
\cdot & {\left[G\left(R x_{3 n+1}, S x_{3 n+1}, I x_{3 n+1}\right)\right.} \\
+ & G\left(Q x_{3 n+1}, J x_{3 n+2}, I x_{3 n+3}\right) \\
+ & \left.G\left(R x_{3 n+1}, S x_{3 n+1}, I x_{3 n+1}\right)\right]^{-1}+B \\
& \cdot G\left(Q x_{3 n+1}, J x_{3 n+2}, I x_{3 n+3}\right)=A \\
& +\left[G\left(R x_{3 n}, S x_{3 n+1}, T x_{3 n+2}\right)\right. \\
& \cdot G\left(R x_{3 n+1}, S x_{3 n+1}, T x_{3 n}\right)
\end{aligned}
$$

$$
\begin{aligned}
& +\left[G\left(R x_{3 n}, S x_{3 n+1}, T x_{3 n+2}\right)\right]^{2} \\
& +G\left(R x_{3 n+1}, S x_{3 n+1}, T x_{3 n}\right) \\
& \left.\cdot G\left(R x_{3 n}, S x_{3 n+1}, T x_{3 n+2}\right)\right] \\
& \cdot\left[G\left(R x_{3 n+1}, S x_{3 n+1}, T x_{3 n}\right)\right. \\
& +G\left(R x_{3 n}, S x_{3 n+1}, T x_{3 n+2}\right) \\
& \left.+G\left(R x_{3 n+1}, S x_{3 n+1}, T x_{3 n}\right)\right]^{-1}+B \\
& \cdot G\left(R x_{3 n}, S x_{3 n+1}, T x_{3 n+2}\right)=A \\
& \cdot G\left(R x_{3 n}, S x_{3 n+1}, T x_{3 n+2}\right)+B \\
& \cdot G\left(R x_{3 n}, S x_{3 n+1}, T x_{3 n+2}\right)=(A+B) \\
& \cdot G\left(R x_{3 n}, S x_{3 n+1}, T x_{3 n+2}\right) .
\end{aligned}
$$

Hence,

$$
\begin{aligned}
& G\left(R x_{3 n+1}, S x_{3 n+2}, T x_{3 n+3}\right) \\
& \quad \leq(A+B) \cdot G\left(R x_{3 n}, S x_{3 n+1}, T x_{3 n+2}\right), \\
& G_{3 n+1} \leq h \cdot G_{3 n},
\end{aligned}
$$

where $h=A+B$. Continuing this procedure, in the end we get

$$
\begin{aligned}
G_{3 n+1} & \leq h \cdot G_{3 n} \leq h^{2} \cdot G_{3 n-1} \leq h^{3} \cdot G_{3 n-2} \leq h^{4} \cdot G_{3 n-3} \\
& \leq \cdots \leq h^{3 n+1} \cdot G_{0} .
\end{aligned}
$$

Clearly, $G_{3 n+1} \rightarrow 0$ as $n \rightarrow \infty$. So, $G\left(R x_{3 n}, S x_{3 n+1}, T x_{3 n+2}\right) \rightarrow$ 0 ; we get the following sequence:

$$
\begin{aligned}
& \left\{R x_{0}, S x_{1}, T x_{2}, R x_{3}, S x_{4}, T x_{5}, R x_{6}, S x_{7}, T x_{8}, \ldots, R x_{3 n+1},\right. \\
& \left.\quad S x_{3 n+2}, T x_{3 n+3}, \ldots\right\},
\end{aligned}
$$

which is a Cauchy sequence in $G$-complete $G$-metric space and therefore converges to a limit point $w$. But all subsequences of a convergent sequence converge; so, we have

$$
\begin{aligned}
\lim _{n \rightarrow \infty} R x_{3 n} & =\lim _{n \rightarrow \infty} Q x_{3 n+1}=w, \\
\lim _{n \rightarrow \infty} S x_{3 n} & =\lim _{n \rightarrow \infty} J x_{3 n+1}=w, \\
\lim _{n \rightarrow \infty} T x_{3 n-1} & =\lim _{n \rightarrow \infty} I x_{3 n}=w .
\end{aligned}
$$

Since $\{S, I\}$ are weakly commuting mappings, thus we have

$$
G\left(S I x_{3 n}, I S x_{3 n}, I S x_{3 n}\right) \leq G\left(I x_{3 n}, S x_{3 n}, S x_{3 n}\right) .
$$

Taking limit $n \rightarrow \infty$ and noting that $S$ and $I$ are continuous mappings, we have

$$
G(S w, I w, I w) \leq G(w, w, w)
$$


which gives the notion that $S w=I w$. Analogously, we can get $T w=J w$ and $R w=Q w$. We claim that $R w \neq S w$ and $S w \neq T w$ and then from condition (3)

$$
\begin{aligned}
& G(R w, S w, T w) \leq A \\
& \quad \cdot[G(R w, S w, T w) G(R w, S w, S w) \\
& \quad+[G(R w, T w, S w)]^{2} \\
& \quad+G(R w, S w, S w) G(R w, T w, S w)] \\
& \quad \cdot(G(R w, S w, S w)+G(R w, T w, S w) \\
& \quad+G(R w, S w, S w))^{-1}+B \cdot G(R w, T w, S w), \\
& G(R w, S w, T w) \leq(A+B) G(R w, T w, S w),
\end{aligned}
$$

which is a contraction:

$$
G(R w, S w, T w)=0 \quad \text { implies } R w=S w=T w .
$$

Similarly, using similar arguments to those given above, we obtain a contradiction for $R w \neq S w$ and $S w=T w$ or for $R w=S w$ and $S w \neq T w$. Hence, in all the cases, we conclude that $R w=S w=T w$. We prove that any fixed point of $R$ is a fixed point of $S, T, Q, I$, and $J$. Assume that $w \in X$ is such that $R w=w$. Now, we prove that $w=T w=S w$. If it is not the case, then, for $w \neq S w$ and $w \neq T w$, we get

$$
\begin{aligned}
& G(w, S w, T w)=G(R w, S w, T w) \leq A \\
& \cdot[G(R w, S w, T w) G(R w, S w, S w) \\
& +[G(R w, T w, S w)]^{2} \\
& \quad+G(R w, S w, S w) G(R w, T w, S w)] \\
& \quad \cdot(G(R w, S w, S w)+G(R w, T w, S w) \\
& \quad+G(R w, S w, S w))^{-1}+B \cdot G(R w, T w, S w), \\
& G(w, S w, T w) \leq(A+B) G(w, T w, S w),
\end{aligned}
$$

where $G(w, S w, T w)=0$ which implies that $w=S w=T w$; in a similar argument, we can prove the other cases.

Uniqueness. Suppose that $S, T, R, I, J$, and $Q$ have two common fixed points $z$ and $w$ such that $z \neq w$. Since condition $G(R x, S x, I x)+G(Q x, J y, I z)+G(R x, S x, I x)=0$ implies $G(S x, T y, R z)+G(Q x, J y, I z)=0$, we have that $G(R z, S z, I z)+$ $G(Q z, J z, I w)+G(R z, S z, I z)=0$ implies $G(S z, T z, R w)+$ $G(Q z, J z, I w)=0$, which can be written as $G(S z, T z, R w)=0$ or $G(Q z, J z, I w)=0$.

Therefore, one can get the following:

$$
G(z, z, w)=0
$$

or $G(z, z, w)=0 \quad$ implies that $z=w$.
Theorem 13 produces the following corollaries.

Corollary 14. Let $(X, G)$ be a $G$-complete $G$-metric space and let $R, S, T, I, J, Q: X \rightarrow X$ be three self-maps and let $\{S, I\}$, $\{T, J\}$, and $\{R, Q\}$ be weakly commuting pairs of self-mapping such that $T(X) \subset I(X), S(X) \subset J(X)$, and $R(X) \subset Q(X)$, satisfying

$$
G(R x, S y, T z) \leq B \cdot G(Q x, J y, I z)
$$

for all $x, y, z$ in $X$ with $x \neq y \neq z \neq x$ with $0 \leq B<1$. Then, $R, S, T, I, J$, and $Q$ have a unique common fixed point in $X$.

Proof. It follows by taking $A=0$ in Theorem 13 .

Corollary 15. Let $(X, G)$ be a $G$-complete $G$-metric space and let $R, S, T, I, J, Q: X \rightarrow X$ be three self-maps and let $\{S, I\}$, $\{T, J\}$, and $\{R, Q\}$ be weakly commuting pairs of self-mapping such that $T(X) \subset I(X), S(X) \subset J(X)$, and $R(X) \subset Q(X)$, satisfying

$$
\begin{aligned}
G( & (R x, S y, T z) \leq A \cdot[G(Q x, S x, I z) G(R x, S x, I x) \\
& +[G(Q x, J y, I z)]^{2} \\
& +G(R x, S x, I x) G(Q x, J y, I z)](G(R x, S x, I x) \\
& +G(Q x, J y, I z)+G(R x, S x, I x))^{-1}+B \\
& +G(R z, T z, S z)
\end{aligned}
$$

for all $x, y, z$ in $X$ with $x \neq y \neq z \neq x A \geq 0$ with $0 \leq$ $A<1, G(R x, S x, I x)+G(Q x, J y, I z)+G(R x, S x, I x) \neq 0$. Then, $R, S, T, I, J$, and $Q$ have a common fixed point. Further, if $G(R x, S x, I x)+G(Q x, J y, I z)+G(R x, S x, I x)=0$ implies $G(S x, T y, R z)+G(Q x, J y, I z)=0$, then $R, S, T, I, J$, and $Q$ have a unique common fixed point in $X$.

Proof. It follows by taking $B=0$ in Theorem 13 .

Corollary 16. Let $(X, G)$ be a $G$-complete $G$-metric space and let $T, R, I, J: X \rightarrow X$ be three self-maps and let $\{T, I\},\{T, J\}$, and $\{R, I\}$ be weakly commuting pairs of self-mapping such that $T(X) \subset I(X), T(X) \subset J(X)$, and $R(X) \subset I(X)$, satisfying

$$
\begin{aligned}
& G(R x, T y, T z) \leq A \cdot[G(I x, T x, I z) G(R x, T x, I x) \\
& \left.\quad+[G(I x, J y, I z)]^{2}+G(R x, T x, I x) G(I x, J y, I z)\right] \\
& \quad \cdot(G(R x, T x, I x)+G(I x, J y, I z) \\
& \quad+G(R x, T x, I x))^{-1}+B \cdot G(I x, J y, I z)
\end{aligned}
$$

for all $x, y, z \in X$ with $x \neq y \neq z \neq x A, B \geq 0$ with $0 \leq A+B<1, G(R x, T x, I x)+G(I x, J y, I z)+G(R x, T x, I x) \neq$ 0 . Then, $T, R, I$, and $J$ have a common fixed point. Further, if $G(R x, T x, I x)+G(I x, J y, I z)+G(R x, T x, I x)=0$ implies $G(S x, T y, R z)+G(I x, J y, I z)=0$, then $T, R, I$, and $J$ have $a$ unique common fixed point in $X$.

Proof. The proof follows by setting $S=T$ and $I=Q$ in Theorem 13. 


\section{Competing Interests}

The authors declare that they have no competing interests.

\section{References}

[1] S. Aleomraninejad, S. Rezapour, and N. Shahzad, "Fixed point results on subgraphs of directed graphs," Mathematical Sciences, vol. 7, article 41, 2013.

[2] K. C. Border, Fixed Point Theorems with Applications to Economics and Game Theory, Cambridge University Press, Cambridge, Uk, 1985.

[3] S. Banach, "Sur les oprations dans les ensembles abstraits et leurs applications aux equations integrales," Fundamenta Mathematicae, vol. 3, pp. 133-181, 1922.

[4] Ya. I. Alber and S. Guerre-Delabriere, "Principle of weakly contractive maps in Hilbert spaces," in New Results in Operator Theory and Its Applications, I. Gohberg and Yu. Lyubich, Eds., vol. 98 of Operator Theory: Advances and Applications, pp. 7-22, Springer, New York, NY, USA, 1997.

[5] R. P. Agarwal, Z. Kadelburg, and S. Radenović, "On coupled fixed point results in asymmetric G-metric spaces," Journal of Inequalities and Applications, vol. 2013, article 528, 2013.

[6] A. Branciari, "A fixed point theorem for mappings satisfying a general contractive condition of integral type," International Journal of Mathematics and Mathematical Sciences, vol. 29, no. 9, pp. 531-536, 2002.

[7] D. S. Jaggi, "Some unique fixed point theorems," Indian Journal of Pure and Applied Mathematics, vol. 8, no. 2, pp. 223-230, 1977.

[8] M. Abbas and T. Nazir, "Fixed points of T-Hardy Rogers type contraction mapping in metric spaces," Afrika Matematika, vol. 25, no. 1, pp. 103-113, 2014.

[9] M. Abbas, T. Nazir, and S. Radenović, "Some periodic point results in generalized metric spaces," Applied Mathematics and Computation, vol. 217, no. 8, pp. 4094-4099, 2010.

[10] M. Abbas, T. Nazir, and S. Radenović, "Common fixed point of generalized weakly contractive maps in partially ordered $G$ metric spaces," Applied Mathematics and Computation, vol. 218, no. 18, pp. 9383-9395, 2012.

[11] S. Radenović, "Remarks on some recent coupled coincidence point results in symmetric G-metric spaces," Journal of Operators, vol. 2013, Article ID 290525, 8 pages, 2013.

[12] B. K. Dass and S. Gupta, "An extension of Banach contraction principle through rational expression," Indian Journal of Pure and Applied Mathematics, vol. 6, no. 12, pp. 1455-1458, 1975.

[13] J. Harjani, B. Lopez, and K. Sadarangani, "A fixed point theorem for mappings satisfying a contractive condition of rational type on a partially ordered metric space," Abstract and Applied Analysis, vol. 2010, Article ID 190701, 8 pages, 2010.

[14] N. V. Luong and N. X. Thuan, "Fixed point theorem for generalized weak contractions satisfying rational expressions in ordered metric spaces," Fixed Point Theory and Applications, vol. 2011, article 46, 2011.

[15] S. Chandok and E. Karapinar, "Common fixed point of generalized rational type contraction mappings in partially ordered metric spaces," Thai Journal of Mathematics, vol. 11, no. 2, pp. 251-260, 2013.

[16] Z. Mustafa, E. Karapinar, and H. Aydi, "A discussion on generalized almost contractions via rational expressions in partially ordered metric spaces," Journal of Inequalities and Applications, vol. 2014, article 219, 2014.
[17] S. Chandok and J. K. Kim, "Fixed point theorem in ordered metric spaces for generalized contractions mappings satisfying rational type expressions," Journal of Nonlinear Functional Analysis and Applications, vol. 17, pp. 301-306, 2012.

[18] S. Chandok, M. S. Khan, and K. P. R. Rao, "Some coupled common fixed point theorems for a pair of mappings satisfying a contractive condition of rational type," Journal of Nonlinear Analysis and Application, vol. 2013, Article ID jnaa-00174, 6 pages, 2013.

[19] D. S. Jaggi and B. K. Dass, "An extension of Banach's fixed point theorem through a rational expression," Bulletin of the Calcutta Mathematical Society, vol. 72, no. 5, pp. 261-262, 1980.

[20] B. G. Pachpatte, "Common fixed-point theorems for mappings satisfying rational inequalities," Indian Journal of Pure and Applied Mathematics, vol. 10, no. 11, pp. 1362-1368, 1979.

[21] Z. Mustafa and B. Sims, "A new approach to generalized metric spaces," Journal of Nonlinear and Convex Analysis, vol. 7, no. 2, pp. 289-297, 2006.

[22] S. Manro, S. S. Bhatia, S. Kumar, and C. Vetro, "A common fixed point theorem for two weakly compatible pairs in $G$ metric spaces using the property E.A," Fixed Point Theory and Applications, vol. 2013, article 41, 2013.

[23] H. K. Nashine and Z. Kadelburg, "Nonlinear generalized cyclic contractions in complete $G$-metric spaces and applications to integral equations," Nonlinear Analysis: Modelling and Control, vol. 18, no. 2, pp. 160-176, 2013.

[24] R. Saadati, S. M. Vaezpour, P. Vetro, and B. E. Rhoades, "Fixed point theorem in generalized partially ordered G-metric spaces," Mathematical and Computer Modelling, vol. 52, no. 5-6, pp. 797-801, 2010.

[25] P. L. Sanodia, D. Jaiswal, and S. S. Rajput, "Fixed point theorems in G-metric spaces via rational type contractive condition," International Journal of Mathematical Archive, vol. 3, no. 3, pp. 1292-1296, 2012.

[26] M. P. Gandhi and K. B. Bajpai, "Unique common fixed point theorem in G-Metric space via rational type contractive condition," in Proceedings of the International Conference on Benchmarks in Engineering Science and Technology, pp. 1-4, September 2012.

[27] R. Shrivastava, M. Sharma, and R. Bhardwaj, "Fixed point results in G-Metric spaces through rational contractive conditions," International Journal of Theoretical and Applied Sciences, vol. 6, pp. 82-88, 2014.

[28] S. Manro, S. S. Bhatia, and S. Kumar, "Expansion mappings theorems in G-metric spaces," International Journal of Contemporary Mathematical Sciences, vol. 5, no. 49-52, pp. 2529-2535, 2010. 


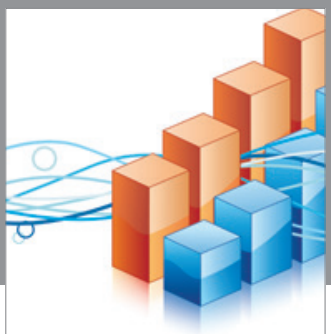

Advances in

Operations Research

vatem alat4

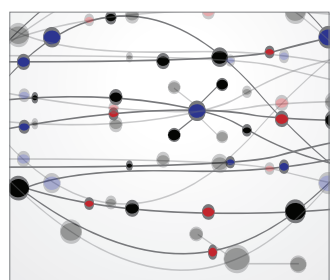

\section{The Scientific} World Journal
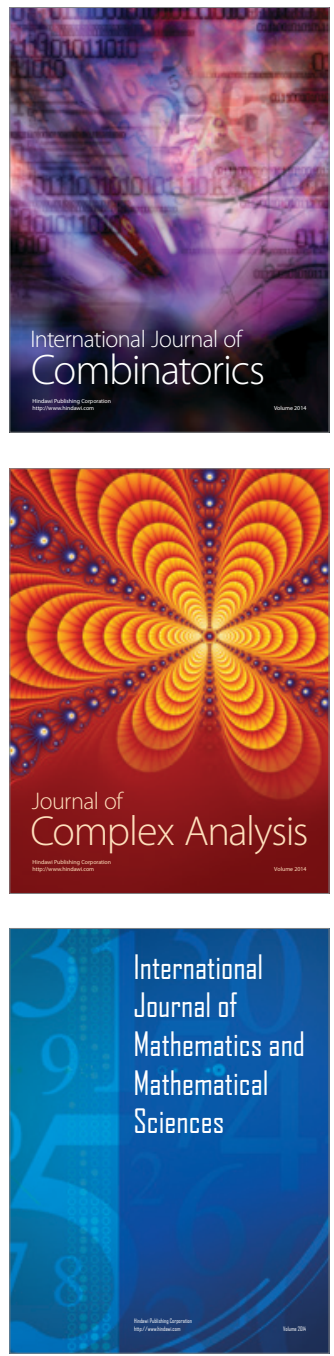
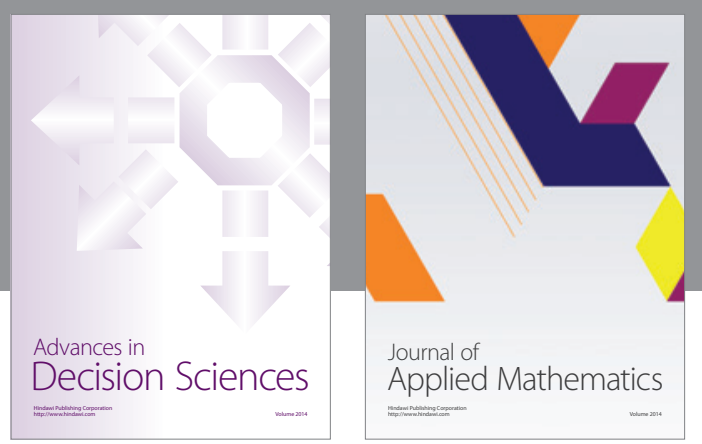

Algebra

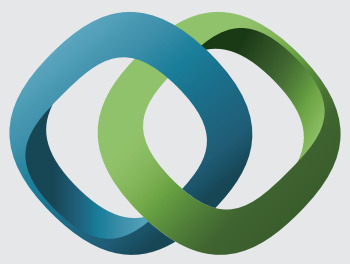

\section{Hindawi}

Submit your manuscripts at

http://www.hindawi.com
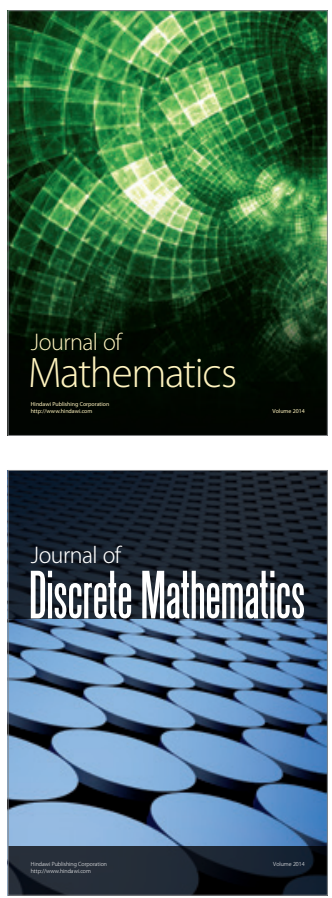

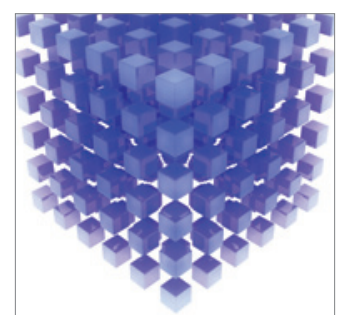

Mathematical Problems in Engineering
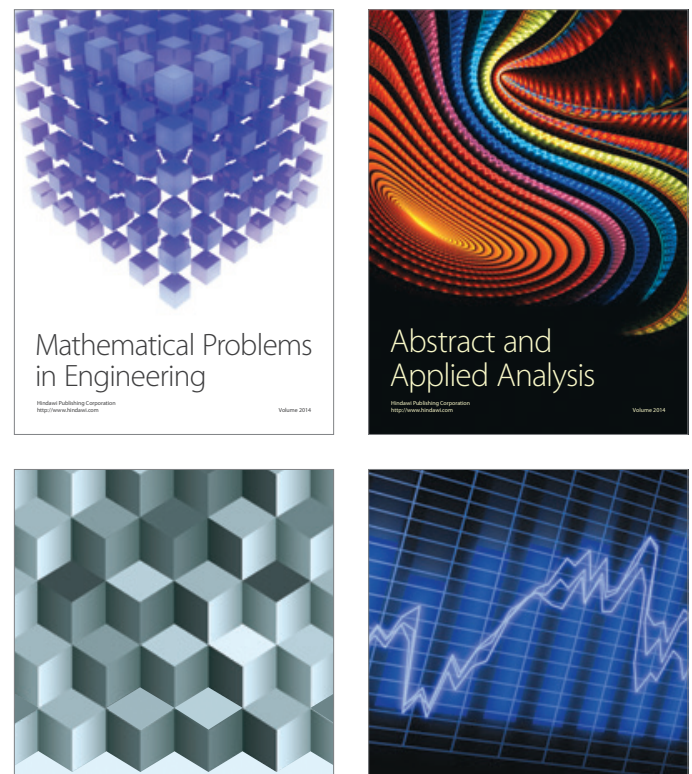

Journal of

Function Spaces

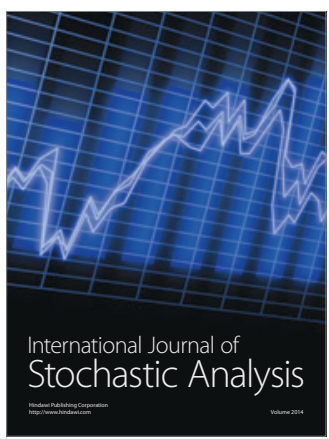

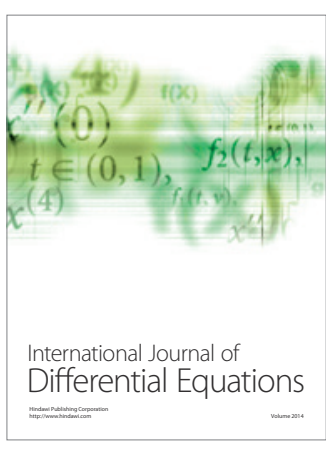
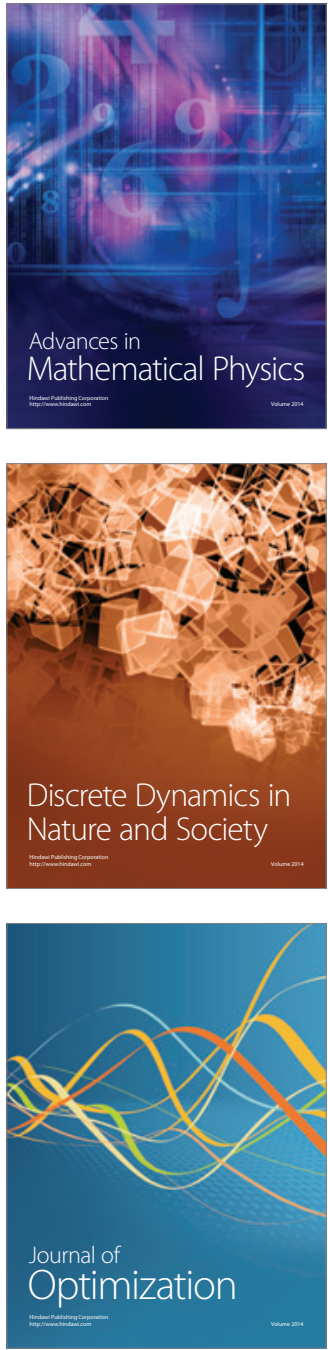